\title{
Frequent epigenetic inactivation of secreted frizzled-related protein 2 (SFRP2) by promoter methylation in human gastric
}

\section{cancer}

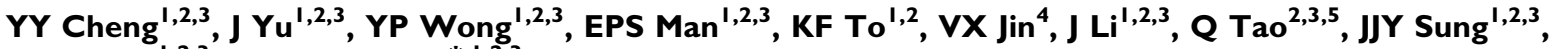 \\ FKL Chan ${ }^{1,2,3}$ and WK Leung ${ }^{*, 1,2,3}$
}

'Institute of Digestive Disease, Faculty of Medicine, The Chinese University of Hong Kong, Hong Kong, China; ${ }^{2}$ State Key Laboratory in Oncology in South China, The Chinese University of Hong Kong, Hong Kong, China; ${ }^{2}$ Li Ka Shing Institute of Health Sciences, The Chinese University of Hong Kong, Hong Kong, China; ${ }^{4}$ Department of Pharmacology and the Genome Center, University of California-Davis, CA, USA; ${ }^{5}$ Department of Clinical Oncology,

The Chinese University of Hong Kong, Hong Kong, China

The role of secreted frizzled-related protein (SFRP) genes in gastric cancer remains largely unknown. We determined the frequency and functional significance of SFRPs hypermethylation in human gastric cancer. The expression and methylation status of four SFRP members (SFRPI, 2, 4, and 5) in primary gastric cancer samples was screened. The biological effects of SFRP were analysed by flow cytometry, cell viability assay and in vivo tumour growth in nude mice. Among the four SFRPs, only SFRP2 was significantly downregulated in gastric cancer as compared to adjacent non-cancer samples $(P<0.01)$. Promoter hypermethylation of SFRP2 was detected in $73.3 \%$ primary gastric cancer tissues, $37.5 \%$ of samples showing intestinal metaplasia and $20 \%$ adjacent normal gastric tissues. Bisulphite DNA sequencing confirmed the densely methylated SFRP2 promoter region. Demethylation treatment restored the expression of SFRP2 in gastric cancer cell lines. Forced expression of SFRP2 induced cell apoptosis, inhibited proliferation of gastric cancer cells and suppressed tumour growth in vivo. Moreover, methylated SFRP2 was detected in 66.7\% of serum samples from cancer patients but not in normal controls. In conclusion, epigenetic inactivation of SFRP2 is a common and early event contributing to gastric carcinogenesis and may be a potential biomarker for gastric cancer.

British Journal of Cancer (2007) 97, 895-901. doi:I0.1038/sj.bjc.6603968 www.bjcancer.com

Published online II September 2007

(c) 2007 Cancer Research UK

Keywords: DNA methylation; gene expression; human gastric cancer; SFRPs

Epigenetic silencing of tumour-related genes due to $\mathrm{CpG}$ island hypermethylation has emerged as one of the most important alternations in gastric cancer development (Leung et al, 2001; Yu et al, 2003). We have previously shown that tumour-related genes including E-cadherin, p15, and p16 were frequently methylated in gastric cancer (Leung et al, 2001) as well as in pre-malignant gastric lesions (Leung et al, 2006), suggesting dysregulation in $\mathrm{CpG}$-island methylation is likely to be involved in the early gastric carcinogenesis process.

Wnt proteins are secreted signalling factors with multiple functions in development and tumourigenesis (Polakis 2007). Wnt binding to frizzled receptors leads to the cytosolic stabilisation and accumulation of $\beta$-catenin, which translocates to the nucleus and forms a complex with $\mathrm{T}$-cell factor transcription factors, thereby regulating target gene expression. Frizzled and secreted frizzled-related proteins (SFRPs), a family of five secreted glycoproteins, are identified as possible negative modulators of the

* Correspondence: Dr WK Leung, Department of Medicine \& Therapeutics, The Chinese University of Hong Kong, Prince of Wales Hospital, Shatin, Hong Kong, China;

E-mail: wkleung@cuhk.edu.hk

Received 3I May 2007; revised I August 2007; accepted 10 August 2007; published online II September 2007
Wnt signal transduction pathway (Heller et al, 2002). The SFRPs are activated through the binding of Wnt proteins to the membrane-bound frizzled receptors, leading to the stabilisation and increase in levels of the transcription factor $\beta$-catenin (Ilyas 2005). Activation of SFRPs results in constitutive activation of Wnt signalling and is associated with inhibition of apoptosis (Melkonyan et al, 1997; Ko et al, 2002), suggesting a critical role of the SFRP genes in the control of Wnt signal pathway. Disruption of this pathway by downregulation of the SFRP genes through promoter methylation has been shown in a few human cancers including colon (Suzuki et al, 2004), bladder (Marsit et al, 2005), oesophagus (Zou et al, 2005), lung (Fukui et al, 2005), and head and neck cancers (Marsit et al, 2006). However, the role Wnt-antagonist genes including SFRP1, SFRP2, SFRP4, and SFRP5 in gastric cancer remains poorly defined. We have previously shown that $S F R P$ was expressed in most of the adjacent non-cancer gastric samples but downregulated in $16 \%$ of gastric cancer samples (To et al, 2001). Moreover, Kirikoshi et al (2001) showed that FZD2, 5, 7, 8, 9, and 10 were upregulated in gastric cancer cell lines.

In the present study, we first determined the expression level of $S F R P$ s in gastric cancer and adjacent non-cancer tissues from gastric cancer patients. Secondly, we examined the methylation status of SFRP genes in gastric cancer and explored the functional significance of methylation-induced silencing of SFRP gene 
expression in gastric cancer cell lines both in vitro and in vivo. We further evaluated the potential use of detecting methylated SFRP DNA in serum as a biomarker for gastric cancer diagnosis.

\section{MATERIALS AND METHODS}

\section{Human gastric samples}

A total of 35 primary gastric adenocarcinomas and their adjacent non-cancer specimens, 24 benign gastric mucosa with intestinal metaplasia, and 10 normal gastric specimens from patients with normal endoscopy were obtained from the Endoscopy Centre of the Prince of Wales Hospital. All specimens were immediately snapped frozen in liquid nitrogen and stored at $-80^{\circ} \mathrm{C}$ until processing. Serum samples were obtained from 18 gastric cancer patients and 18 normal controls without gastric cancer. All subjects gave informed consent for obtaining the study materials. The study protocol was approved by the Clinical Research Ethics Committee of the Chinese University of Hong Kong.

\section{Cell lines and cell culture}

A total of seven gastric cancer cell lines (Kato III, MKN-28, MKN45, SNU-1, SNU-16, AGS, and NCI-N87) were obtained from Riken Gene Bank (Japan) and American Type Culture Collection (Manassas, VA, USA). Cells were cultured in RPMI 1640 (Invitrogen, Carlsbad, CA, USA) containing 10\% fetal bovine serum at $5 \% \mathrm{CO}_{2}, 37^{\circ} \mathrm{C}$, and $95 \%$ humidity.

\section{Reverse transcription, polymerase chain reaction (RT - PCR) and real-time RT - PCR}

Since SFRP3 does not contain a CpG island of more than $200 \mathrm{bp}$, we only examined the gene expression levels of SFRP1, SFRP2, SFRP4, and SFRP5 in this study. Total RNA was extracted from 14 pairs of gastric cancer specimens and 7 gastric cancer cell lines by Trizol reagent (Gibco BRL, Life Technologies, Invitrogen) according to the manufacturer's instruction. Reverse transcription reaction was performed using $2 \mu \mathrm{g}$ of total RNA with a first strand cDNA kit (Promega, Madison, WI, USA). The mRNA expression levels of the SFRPs were determined by PCR (PTC-200, MJ Research, Waltham, MA, USA) and quantitative real-time PCR (LightCycler, Roche Diagnostic, Germany). Probe Design software (Roche Diagnostic) was used for designing PCR primers (Table 1A). Glyceraldehyde-3-phosohate dehydrogenase (GAPDH) was used as an internal control of RNA integrity. The ratio of SFRPs to GAPDH expression was determined by Relative Quantification Software (Roche Diagnostic).

\section{Bisulphite treatment of DNA, methylation-specific polymerase chain reaction (MSP) and quantitative methylation-specific PCR}

Genomic DNA was extracted from 30 pairs of gastric cancer tissues, 24 benign gastric mucosa with intestinal metaplasia, and 10 normal gastric specimens or cell lines by a DNA mini kit (Qiagen, Valencia, CA, USA). Methylation statuses of SFRP1, SFRP2, SFRP4, and SFRP5 in gastric tissues and cell lines were determined by MSP. Briefly, $2 \mu \mathrm{g}$ of genomic DNA was bisulphite-treated with Zymo DNA Modification Kit (Zymo Research, Orange, CA, USA). Bisulphite-treated DNA was used as a template for MSP and quantitative PCR by ABI 2700 thermocycler (Applied Biosystems, CA, USA) and LightCycler (Roche Diagnostic), respectively. OLIGO 6 software (Molecular Biology Insights, CO, USA) was used for designing primers specific for methylated and unmethylated alleles (Table 1B). CpGenome ${ }^{\mathrm{TM}}$ universal methylated DNA (Chemicon International Inc., CA, USA) was used as positive control for methylation and water was used as a negative control
Table I List of primer sequences

\begin{tabular}{ll}
\hline (A) Primer sequences for RT-PCR \\
SFRPI \\
Sense & 5'-CCAGCGAGTACGACTACGTGAGCTT-3' \\
Anti-sense & 5'-CTCAGATTTCAACTCGTTGTCACAGG-3' \\
SFRP2 & \\
Sense & 5'-GATGATGACAACGACATAATGGAAACG-3' \\
Anti-sense & 5'-GAGTGTGCTTGGGGAACGGGAGCT-3' \\
SFRP4 & \\
Sense & 5'-GGTACAGGAAAGGCCTCTTGATGTTG-3' \\
Anti-sense & 5'-GGATCTTTTACTAAGCTGATCTCTCC-3' \\
SFRP5 & \\
Sense & 5'-TGCGCCCAGTGTGAGATGGAGCAC-3' \\
Anti-sense & 5'-CCCATCCCTTAGGCCTTGTGCCAGT-3' \\
GAPDH & \\
Sense & 5'-GGAGTCAACGGATTTGGT-3' \\
Anti-sense & 5'-GTGATGGGATTTCCATTGAT-3'
\end{tabular}

(B) Primer sequences for MSP

SFRP

Methylated sense 5'-TGTAGTITTCGGAGTTAGTGTCGCGC-3' Methylated 5'-CCTACGATCGAAAACGACGCGAACG-3'

anti-sense

Unmethylated 5'-GTITGTAGTIITGGAGTTAGTGTTGTGT-3'

sense

Unmethylated $\quad 5^{\prime}$-CTCAACCTACAATCAAAAACAACACAAACA-3'

anti-sense

SFRP2

Methylated sense 5'-GGGTCGGAGTTTTTCGGAGTTGCGC-3' Methylated 5'-CCGCTCTCTTCGCTAAATACGACTCG-3'

anti-sense

Unmethylated sense $5^{\prime}$-गITTGGGTTGGAGTIIITGGAGTTGTGT-3'

Unmethylated 5'-AACCCACTCTCTTCACTAAATACAACTCA-3' anti-sense

SFRP4

Methylated sense 5'-GGGTGATGTTATCGTTITTGTATCGAC-3'

Methylated 5'-CCTCCCCTAACGTAAACTCGAAACG-3'

anti-sense

Unmethylated 5'-GGGGGTGATGTTATTGTTTTGTATTGAT-3'

sense

Unmethylated $\quad$ 5'-CACCTCCCCTAACATAAACTCAAAACA-3'

anti-sense

SFRP5

Methylated sense 5'-AAGATTTGGCGTTGGGCGGGACGTTC-3'

Methylated 5'-ACTCCAACCCGAACCTCGCCGTACG-3'

anti-sense

Unmethylated 5'-GTAAGATTTGGTGTTGGGTGGGATGTT-3'

sense

Unmethylated $\quad 5^{\prime}$-AAAACTCCAACCCAAACCTCACCATACA-3

anti-sense

$\beta$-actin

Sense 5'-TGGTGATGGAGGAGGTTTAGTAAGT-3'

Anti-sense 5'-AACCAATAAAACCTACTCCTCCCTTAA-3'

(C) Primer sequences for bisulphite DNA sequencing

SFRPI

Sense

Anti-sense

SFRP2

Sense

Anti-sense

SFRP4

Sense

Anti-sense

SFRP5

Sense

Anti-sense

5'-TGGTTITGTIIITTAAGGGGTGTTGAGT-3'

5'-TCCTACCRCAAACTTCCAAAAACCTCC-3'

5'-AATTAGATTTAGAAAGTAGTGATTAGT-3'

5'-AACCAAAACCCTACAACATCGTAAAC-3'

5'-GAGGGGGTGATGTTATYGTIITTGTAT-3'

5'-CCCCAAACTCCAATCRACAACAAAAC-3'

5'-GGGAGGTAGGGAGTTITGGGGAGAA-3'

5'-CCCAAATAAATAACAACCTACRCTAC-3'

Abbreviations: $R T-P C R=$ reverse transcription $-P C R ; S F R P=$ secreted frizzled related protein; GAPDH = glyceraldehyde-3-phosohate dehydrogenase; $M S P=$ methylation-specific polymerase chain reaction.

for each amplification. To quantify the amount of DNA methylation, the methylated DNA was determined by the threshold cycle number for each sample against a standard curve generated by 
CpGenome universal methylated DNA (Chemicon, Temecula, CA, USA). The results were expressed as the ratio of methylated DNA to that of $\beta$-actin. Samples with a ratio of $>0.5$ were considered to have a high level of DNA methylation (Chan et al, 2005).

\section{Bisulphite sequencing}

For bisulphite DNA genomic sequencing, $2 \mu \mathrm{l}$ of bisulphite-treated DNA was amplified using primers list in Table 1C. The PCR products were electrophoresed and cloned into the pCR2.1-TOPO cloning vector (Invitrogen). A total of 8-10 colonies were randomly chosen for plasmid DNA extraction with Qiaprep Spin Mini kit (Qiagen). Plasmid DNA was sequenced using the ABI PRISM BigDye Terminator Cycle Sequencing Kit in ABI 3100 sequencer (Applied Biosystems). Sequencing analysis was performed by SeqScape software (Applied Biosystems).

\section{Construction of expression plasmids}

The full-length pcDNA3.1 $(+) S F R P 2$ clone was made by cloning of the full-length PCR product of SFRP2 with PFU DNA polymerase (Invitrogen). All the plasmid sequences and orientations were confirmed by DNA sequencing.

\section{Western blot}

A total of $20 \mu \mathrm{g}$ of protein of each sample was loaded in $12.5 \%$ sodium dodecyl sulfate/polyacrylamide gel electrophoresis. The proteins on the polyacrylamide gel were transferred to a member for antibody incubation. Polyclonal SFRP2 antibody (Santa Cruz Biotechnology, Santa Cruz, CA, USA) and the corresponding secondary antibody (Santa Cruz Biotechnology) were applied before immunoblotting. Immunoblots were developed by using the enhanced chemiluminescence detection system (Amersham, Piscataway, NJ, USA) according to the manufacturer's protocol. Human $\beta$-actin was used as the control of protein integrity.

\section{Cell proliferation assay}

Transiently transfected MKN45 cells with or without SFRP2 expression vector were selected for proliferation assay. Cells were seeded in 96-well plates. The colorimetric MTs (Promega) assay was used to measure cell numbers at 24 and $48 \mathrm{~h}$. Experiment was performed in triplicates.

\section{Flow cytometric (FCM) detection}

Cell cycle analysis was carried out by FCM. Briefly, cells transfected with or without SFRP2 were removed and washed twice with PBS at 24 and $48 \mathrm{~h}$. The cells were then fixed in ice-cold ethanol for $1 \mathrm{~h}$. The samples were concentrated by removing the ethanol and treated with $0.01 \%$ RNase $\left(10 \mathrm{mg} \mathrm{ml}^{-1}\right.$, Sigma, St Louis, Mo, USA) for $10 \mathrm{~min}$ at $37^{\circ} \mathrm{C}$. Cellular DNA was stained with $0.05 \%$ propidium iodide for $20 \mathrm{~min}$ at $4^{\circ} \mathrm{C}$ in dark. The cell cycle distribution was determined using a FACScan flow cytometer (Becton Dickinson, Mountain View, CA, USA) and 10000 cells were analysed with MultiCycle software package (Phoenix, San Diego, CA, USA).

\section{Nude mice assay}

Transiently transfected SFRP2/MKN45 cells, mock-transfected cells and parental MKN45 cells were re-suspended in fresh PBS and counted. MKN45 $\left(1 \times 10^{6}\right.$ cells $)$ with or without SFRP2 expression vector was injected subcutaneously into the dorsal flank of nude mice (five per group). The time interval of tumour occurrence and the dimension of the tumour were recorded every 3 days until the end of week 3. The animal study protocol was approved by the
Animal Experimentation Ethics Committee of the Chinese University of Hong Kong and all procedures were performed in compliance to the guidelines of the United Kingdom Co-ordinating Committee on Cancer Research.

Two perpendicular tumour diameters, width and length, were obtained with calipers and used to calculate tumour volume with the following formula: tumour volume $=$ length $\times$ width $^{2} \times 0.52$ (Yagi and Bekesi, 1990). The experiment was repeated three times.

\section{Statistical analysis}

The results were expressed as mean \pm s.d. or percentage where appropriate. Mann-Whitney $U$-test was used to compare the two sample groups including in vivo tumour growth, percentage of methylation, age, sex, and the difference in mRNA levels between cancer and non-cancer specimens. Student's $t$-test was used to compare the differences of SFRP2 expression on the effect of cell apoptosis and cell proliferation. $\chi^{2}$ test was used for comparison of methylation frequency and Helicobacter pylori infection. All statistical calculations were done using SPSS version 11.0 for windows (SPSS Inc., Chicago, IL, USA). A $P$-value of $<0.05$ was taken as statistical significance.

\section{RESULTS}

Gene silencing and methylation status of SFRPs in primary human gastric cancers

We determined the mRNA expression of SFRPs in 14 pairs of primary human gastric cancers and adjacent non-cancerous tissues by quantitative RT-PCR. When compared to adjacent non-cancer tissues, SFRP2 was found to be significantly downregulated in primary gastric tumour specimens (Figure 1). However, this difference was not seen in SFRP1, SFRP4, and SFRP5.

To investigate the role of promoter methylation in silencing of SFRPs in human gastric cancers, the methylation status of SFRPs was studied in 30-paired gastric cancer specimens and their noncancer tissue by MSP (Table 2). For SFRP2, 22 (73.3\%) gastric cancer specimens had SFRP2 methylation, which was significantly higher than that of adjacent non-cancer tissue $(20 \%, P<0.0001)$ (Table 2). There was also no significant difference in the methylation status between cancer and adjacent non-cancer areas in other SFRPs. Notably, SFRPs methylation was not seen in gastric specimens from 10 controls without gastric cancer. In addition, quantitative MSP was performed to quantify the degree of

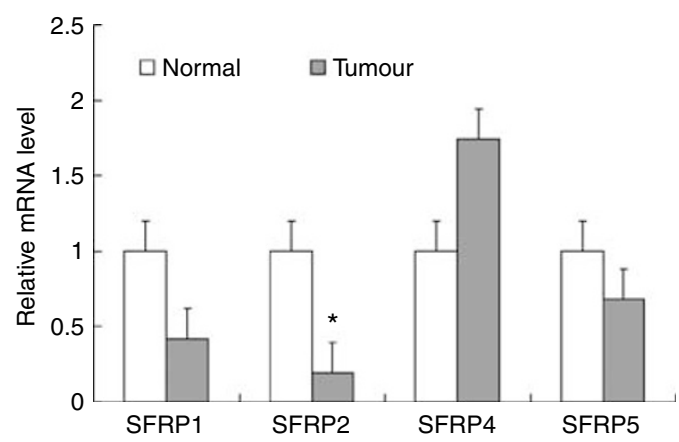

Figure I mRNA expression levels of secreted frizzled-related proteins (SFRPs) in primary gastric cancer (Tumour) and their adjacent non-cancer tissues (Normal) as determined by quantitative real-time PCR. The results were expressed as the ratio of copies of target gene relevant to GAPDH form three independent experiments $(n=\mid 4)$. Data are expressed as mean \pm s.d., $* P<0.01$ 
Table 2 Frequency of SFRPs methylation in primary gastric samples

\begin{tabular}{|c|c|c|c|c|c|c|c|}
\hline & \multicolumn{3}{|c|}{ Adjacent non-tumour tissues } & \multicolumn{4}{|c|}{ Tumour } \\
\hline & No. & Methylated & $\%$ & No. & Methylated & $\%$ & $P$-value \\
\hline SFRPI & 30 & 25 & 83.3 & 30 & 27 & 90 & $>0.05$ \\
\hline SFRP2 & 30 & 6 & 20 & 30 & 22 & 73.3 & $<0.000 \mid$ \\
\hline SFRP4 & 30 & 6 & 20 & 30 & 9 & 30 & $>0.05$ \\
\hline SFRP5 & 30 & 15 & 50 & 30 & 9 & 30 & $>0.05$ \\
\hline
\end{tabular}

Abbreviation: SFRPs $=$ secreted frizzled-related proteins.

Table 3 Patients' characteristics and methylation status

\begin{tabular}{|c|c|c|c|c|c|c|c|c|c|c|c|c|}
\hline \multirow[b]{2}{*}{ Patient } & \multirow[b]{2}{*}{ Sex } & \multirow{2}{*}{\multicolumn{2}{|c|}{ Age type }} & \multirow{2}{*}{ H. pylori } & \multicolumn{2}{|c|}{ SFRP I } & \multicolumn{2}{|c|}{ SFRP2 } & \multicolumn{2}{|c|}{ SFRP4 } & \multicolumn{2}{|c|}{ SFRP5 } \\
\hline & & & & & $\mathbf{N}$ & $\mathbf{T}$ & $\mathbf{N}$ & $\mathbf{T}$ & $\mathbf{N}$ & $\mathbf{T}$ & & $\mathbf{T}$ \\
\hline 1 & $\mathrm{~F}$ & 78 & Mix & Pos & + & + & - & + & - & - & + & - \\
\hline 2 & $M$ & 70 & - & - & + & + & - & + & + & + & + & - \\
\hline 3 & $M$ & 54 & Int & Pos & + & + & - & + & - & - & + & + \\
\hline 4 & $\mathrm{~F}$ & 77 & Diff & Pos & + & + & - & + & - & - & - & - \\
\hline 5 & $\mathrm{~F}$ & 83 & - & - & + & + & - & + & - & + & + & - \\
\hline 6 & $M$ & 73 & Diff & $\mathrm{Neg}$ & + & + & + & + & - & - & + & - \\
\hline 7 & $M$ & 66 & Diff & $\mathrm{Neg}$ & + & - & + & + & - & - & + & - \\
\hline 8 & $\mathrm{~F}$ & 81 & Diff & $\mathrm{Neg}$ & + & _- & - & _- & - & - & + & + \\
\hline 9 & $M$ & 42 & Diff & $\mathrm{Neg}$ & - & + & - & + & - & - & - & + \\
\hline 10 & $M$ & 48 & - & - & + & + & - & + & - & - & + & + \\
\hline 11 & $M$ & 79 & Int & $\mathrm{Neg}$ & + & + & + & - & + & - & + & + \\
\hline 12 & $\mathrm{~F}$ & 72 & - & - & + & + & + & + & + & + & - & + \\
\hline 13 & $\mathrm{~F}$ & 86 & Int & Pos & + & + & + & _- & + & - & + & + \\
\hline 14 & $M$ & 69 & Int & $\mathrm{Neg}$ & + & + & - & + & - & + & - & + \\
\hline 15 & $\mathrm{~F}$ & 69 & Mix & Pos & - & + & - & + & - & - & - & - \\
\hline 16 & $M$ & 58 & - & Pos & + & - & - & + & - & - & - & - \\
\hline 17 & $M$ & 50 & Diff & neg & + & + & - & - & - & - & + & + \\
\hline 18 & $M$ & 42 & Int & Pos & + & + & - & - & - & - & + & + \\
\hline 19 & $\mathrm{~F}$ & 80 & Int & Pos & + & + & - & - & - & - & - & + \\
\hline 20 & $M$ & 77 & - & - & _- & + & - & + & - & - & - & - \\
\hline 21 & $M$ & 39 & Int & $\mathrm{Neg}$ & + & + & - & + & - & + & + & + \\
\hline 22 & $\mathrm{~F}$ & 32 & Diff & Neg & - & + & - & - & - & - & - & + \\
\hline 23 & $M$ & 62 & Int & Pos & + & + & - & + & - & + & - & + \\
\hline 24 & $M$ & 49 & Int & Pos & + & + & - & + & - & + & - & + \\
\hline 25 & $M$ & 57 & Int & Neg & + & + & - & - & + & - & + & + \\
\hline 26 & $M$ & 77 & Int & Pos & + & + & + & + & + & - & - & + \\
\hline 27 & $M$ & 70 & Int & Neg & - & + & - & + & - & + & - & + \\
\hline 28 & $\mathrm{~F}$ & 61 & Diff & Pos & + & + & _- & + & - & - & + & + \\
\hline 29 & $M$ & 76 & Int & Pos & + & + & - & + & - & + & - & + \\
\hline 30 & $\mathrm{~F}$ & 73 & Mix & $\mathrm{Neg}$ & + & + & - & + & - & - & - & + \\
\hline
\end{tabular}

Abbreviations: SFRPs = the role of secreted frizzled-related proteins; $T=$ tumour $\mathrm{N}=$ adjacent non-tumour, Int = intestinal; Diff = diffuse; Pos = positive; Neg = negative; + = methylation.

methylation in primary gastric cancer. Consistent with the semiquantitative MSP results, the mean methylation level of SFRP2 was significantly higher in cancer than in adjacent non-cancer specimen $(P<0.001)$. We also determined the correlation between $H$. pylori infection and SFRP2 methylation. H. pylori infection was detected in $59 \%$ of gastric cancer tissues with methylation in SFRP2 gene and $43 \%$ of gastric cancer tissues without SFRP2 methylation $(P=0.69)$. There was no association between promoter hypermethylation of SFRP2 gene and the status of $H$. pylori infection. In addition, there was no significant association between SFRP2 methylation and the clinico-pathological characteristics of cancer including age, sex, tumour type, tumour differentiation, and prognosis for patients (Table 3).

\section{SFRP2 expression could be restored with $5^{\prime} \mathrm{Aza}-\mathrm{dC}$ treatment in gastric cancer cell lines}

As shown in Figure 2A, SFRP2 was silenced in all seven human gastric cancer cell lines. We examined the role of methylation in the silencing of SFRP2. Using MSP, SFRP2 methylation was
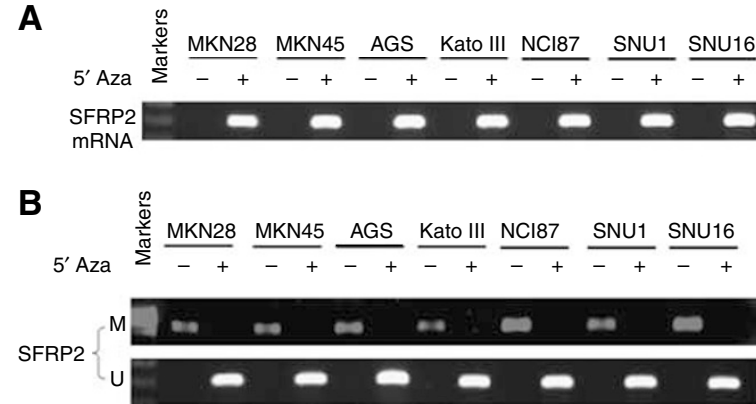

Figure 2 (A) The mRNA expression of secreted frizzled-related protein 2 (SFRP2) in gastric cancer cell lines (MKN28, MKN45, AGS, Kato III, NCl87, SNUI, and SNUI6) treated with or without demethylation agent $5^{\prime} \mathrm{Aza}-$ DC as determined by RT-PCR. Pharmacologic treatment with $5^{\prime}$ Aza-DC $\left(5^{\prime} \mathrm{Aza}\right)$ restored the expression of SFRPs in tumour cell lines. (B) The methylation status of SFRP2 in gastric cancer cell lines treated with or without $5^{\prime}$-Aza-DC as determined by methylation specific PCR. M: methylated primers; U: unmethylated primers.

detected in all these seven cell lines with silenced expression (Figure 2B). To confirm that $\mathrm{CpG}$ methylation is indeed responsible for the silencing of SFRP2, we treated these heavily methylated and silenced cell lines with 5-Aza-DC, a methyltransferase inhibitor. SFRP2 expression was markedly induced after the treatment in all the cell lines (Figure 2A). These results demonstrate that $\mathrm{CpG}$ methylation directly contributes to the silencing of SFRP2 in tumour cells.

\section{Bisulphite sequencing of SFRP2}

To verify the MSP findings and to study the extent of promoter methylation of $S F R P 2$, we performed high-resolution bisulphite genomic sequencing in the seven cell lines, four gastric cancers with low or silenced SFRP2 expression, and in their adjacent noncancer tissues. The area of the $\mathrm{CpG}$-rich region around the transcription initiation site of SFRP2 gene between the nucleotides -362 and +9 which spanned $34 \mathrm{CpG}$ site was sequenced (Figure 3). There was extensive methylation in the promoter region of the MKN28 cell lines and gastric cancer tissue with low or silenced SFRP2 expression, whereas a very few methylated CpG sites were detected in adjacent non-cancer gastric tissues. Demethylation treatment slightly reduced the methylation density in MKN28 cells.

\section{SFRP2 inhibits tumour cell proliferation and induces cell apoptosis in vitro}

The frequent silencing of SFRP2 by methylation in gastric cancer but not in adjacent gastric mucosa suggests a potential tumour suppressor role of this gene. To test this speculation, we examined the effect of $S F R P 2$ transfection on cell proliferation and apoptosis of gastric cancer cells. SFRP2 expression vector was transiently transfected into MKN45 cell line with complete methylation and silencing of SFRP2. Forced expression of SFRP2 in transfected MKN45/SFRP2 cells was confirmed by RT - PCR (Figure 4A) and western blot (Figure $4 \mathrm{~B}$ ). After $48 \mathrm{~h}$ of transfection, cell viability was reduced from 0.77 to $0.56 \quad(P<0.001)$ (Figure $4 \mathrm{C}$ ) as determined by cell proliferation assay. Cell apoptosis as determined by sub- $G_{1}$ phase cells was significantly induced from 0.12 to $3.79 \%$ at $24 \mathrm{~h}(P<0.05)$ and from 0.56 to $8.1 \%$ at $48 \mathrm{~h}(P<0.001)$ (Figure 5).

\section{SFRP2 inhibits gastric tumour growth in vivo}

In light of the observed anti-proliferative and pro-apoptotic effects of SFRP2 on MKN45 in vitro, we tested whether SFRP2 would alter 


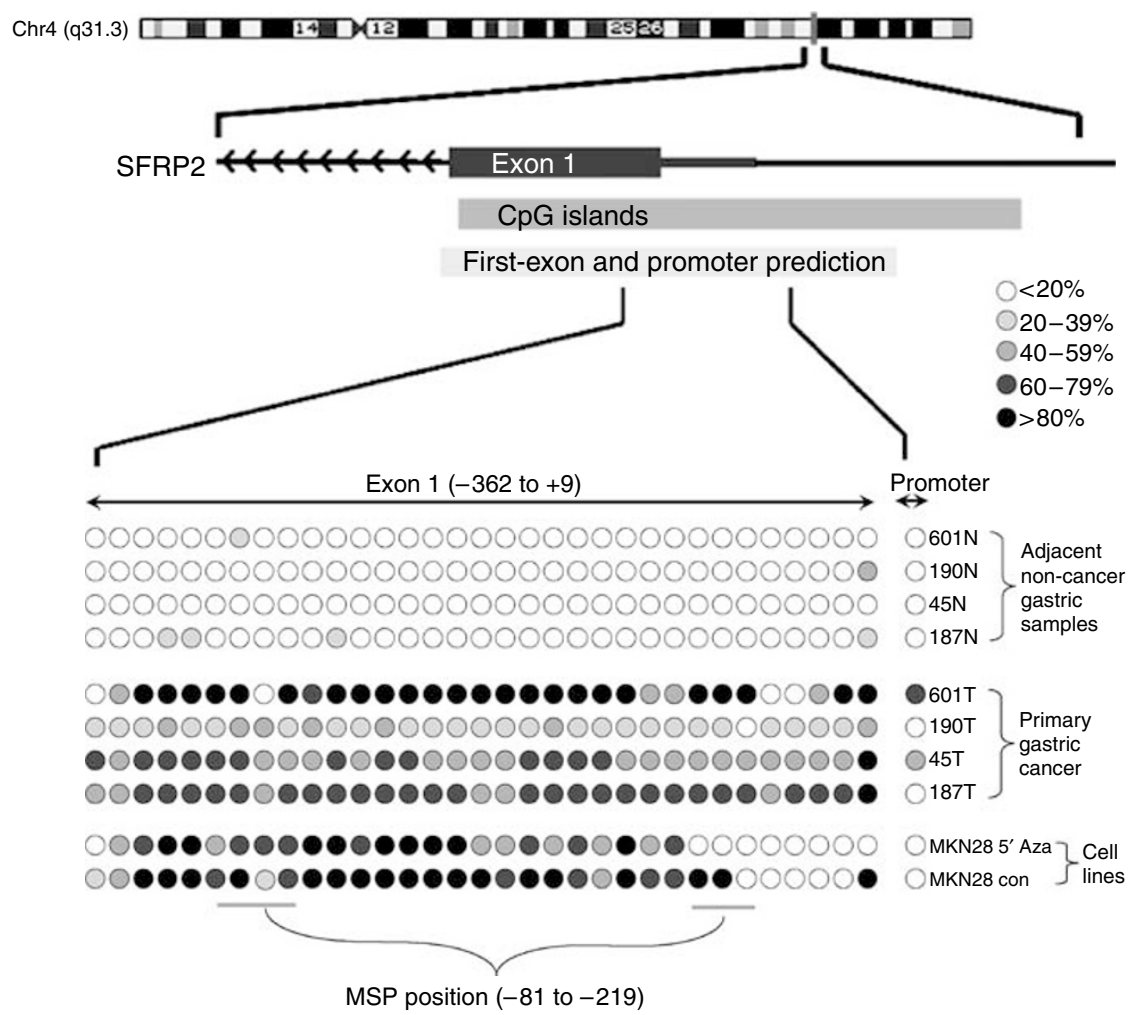

Figure 3 A representative picture of bisulphite sequencing in secreted frizzled-related protein 2 (SFRP2) gene. The CpG island, MSP region ( -219 to $-8 \mathrm{I})$ and bisulphite sequence region $(-362$ to +9$)$ were shown in the upper panel. A 37I-bp region with 34 CpG site was analysed. Each row of CpG sites represented an individual allele of the SFRP2 promoter analysed. Percentage methylation was determined as percentage of methylated cytosine from 8 to 10 randomly sequenced colonies.

the growth of gastric cancer cells in vivo (Figure 6A). The tumour growth curve of transiently transfected MKN45/3.1-vector control and MKN45/SFRP2 expression vector in nude mice was shown in Figure 6B. The mean tumour volume was significantly smaller in SFRP2-transfected nude mice as compared to the nude mice with MKN45/3.1-vector control $(P<0.0001)$.

\section{Methylation of SFRP2 in pre-neoplastic gastric lesion}

To determine the onset time of SFRP2 methylation in the gastric carcinogenesis process, we examined endoscopic gastric specimens containing foci of intestinal metaplasia, a pre-neoplastic lesion, from patients without gastric cancer. Among the 24 samples with intestinal metaplasia, $9(37.5 \%)$ had promoter methylation in SFRP2 detected.

\section{Methylation of SFRP2 in serum samples of gastric cancer patients}

To further investigate whether SFRP2 methylation could be used as a biomarker for gastric cancer, serum samples of gastric cancer patients were tested for methylated SFRP2 DNA. A total of 12 of 18 (66.7\%) serum samples from cancer patients had SFRP2 methylation detected whereas SFRP2 methylation was not detected in the serum of 18 normal subjects.

\section{DISCUSSION}

The inappropriate activation of Wnt signalling through mutation of $\beta$-catenin or APC and/or downregulation of negative regulators such as SFRPs occurs frequently in cancers. The function of SFRPS as antagonist of the Wnt pathway provides a potential mechanism to suppress the abnormal activation of this pathway. Wnt signal activation by mutant $\beta$-catenin or APC could be suppressed partially by overexpression of SFRPs (Suzuki et al, 2002, 2004). Our previous study has shown that SFRP was downregulated in gastric cancer (To et al, 2001) and may carry a tumour suppressor potential. To address whether epigenetic mechanisms were responsible for SFRP downregulation, we extend the study to determine the mRNA expression level and the methylation status of SFRPs in gastric cancer. Among the four SFRPs with CpG islands in the promoter region, we found that only $S F R P 2$ was silenced and methylated in gastric cancer. Promoter methylation of SFRP2 was specifically associated with low or absent mRNA expression in gastric cancer. The CpG island methylation status of SFRP2 was further confirmed by bisulphite sequencing. In addition, we confirmed that the SFRP2 expression was restored after the demethylating agent, 5-aza- $2^{\prime}$-deoxycytidine, treatment in gastric cancer cell lines. These data suggested that hypermethylation of the promoter region might be critical for the transcriptional silencing of $S F R P 2$ in gastric cancers.

The functional role of $S F R P 2$ in gastric cancer cells remains unclear and was further evaluated by examining the inhibitory effect of SFRP2 expression on tumour cells. Forced expression of $S F R P 2$ in gastric cancer cell line MKN45 with hypermethylated SFRP2 inhibits cell proliferation, induces cell apoptosis, and inhibits in vivo tumour growth. Consistent with these findings, Suzuki et al (2004) demonstrated that overexpressed SFRPs reduced colony formation and induced apoptosis in colon cancer cells. Taken together, these data highly suggest that SFRP2 acts as a functional tumour suppressor gene in gastric cancer and its silencing may enhance tumour growth and expansion. 
A
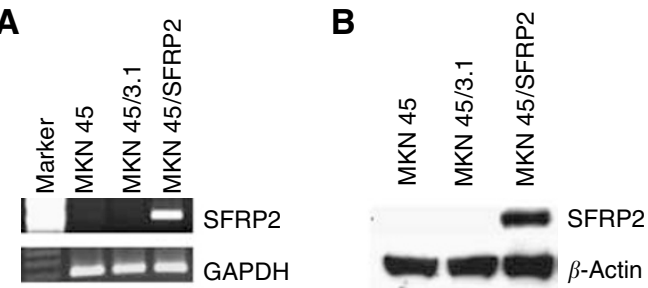

C 1.00

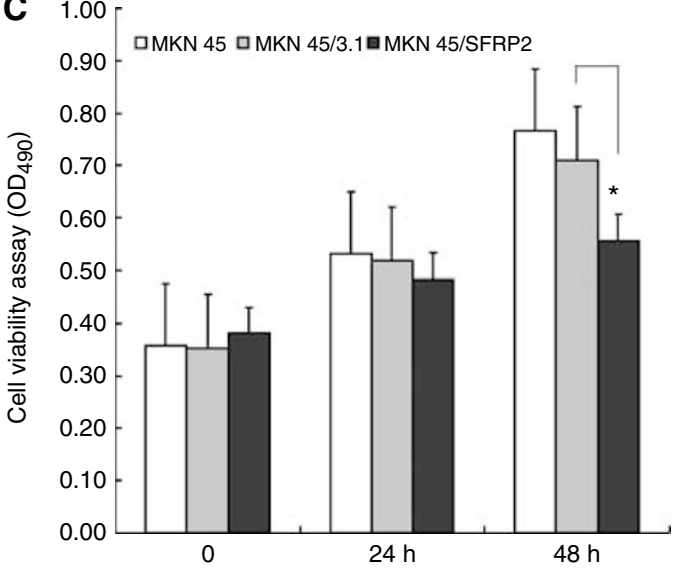

Figure 4 Transient transfection of secreted frizzled-related protein 2 (SFRP2) gene in MKN45 cell lines. (A) Strong SFRP2 mRNA expression was observed in MKN45 cells transfected with pCDNA3.I $(+)$ SFRP2, but not in cells transfected with the empty vector. (B) Strong SFRP2 protein expression was observed in MKN45 cells transfected with PCDNA3.I + SFRP2, but not in cells with empty vector of in parental MKN45. (C) SFRP2 significantly suppressed cell viability at $48 \mathrm{~h}$. Values are expressed as the mean \pm s.d. from three independent experiments $(* P<0.00$ I) .

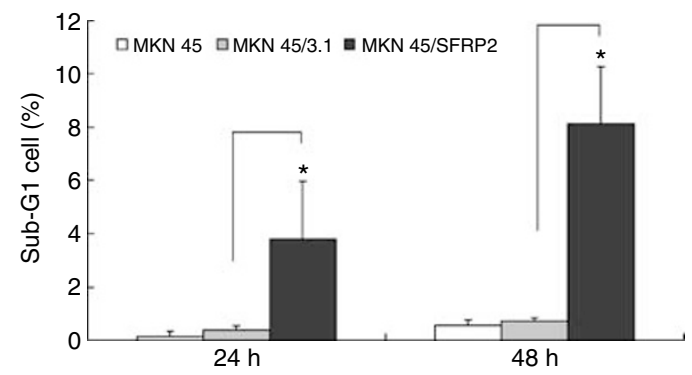

Figure 5 Effect of SFRP2 on apoptosis of human gastric cancer cells. SFRP2 increased the rate of apoptosis in MKN45 cells transfected with PCDNA3.I (+)SFRP2, as determined by the number of cells with sub-G, DNA content by flow cytometry. Values are expressed as the mean \pm s.d. of three replicate experiments. $* P<0.001$

Recent epigenetic studies have suggested that silencing of the genes by DNA hypermethylation at CpG islands tended to be accumulated in the multi-step pathway of gastric carcinogenesis (Kang et al, 2001; To et al, 2002; Waki et al, 2002). Promoter methylation of tumour suppressor genes has been detected in early stages of gastric cancer development (Tamura 2002; Waki et al, 2002). In this study, methylation of SFRP2 was detected in intestinal metaplasia, a pre-neoplastic gastric lesion, which implicates their early involvement in the multi-step gastric carcinogenesis process. Our results showed that the frequency of hypermethylation decreased from $73.3 \%$ in gastric cancer to $37.5 \%$
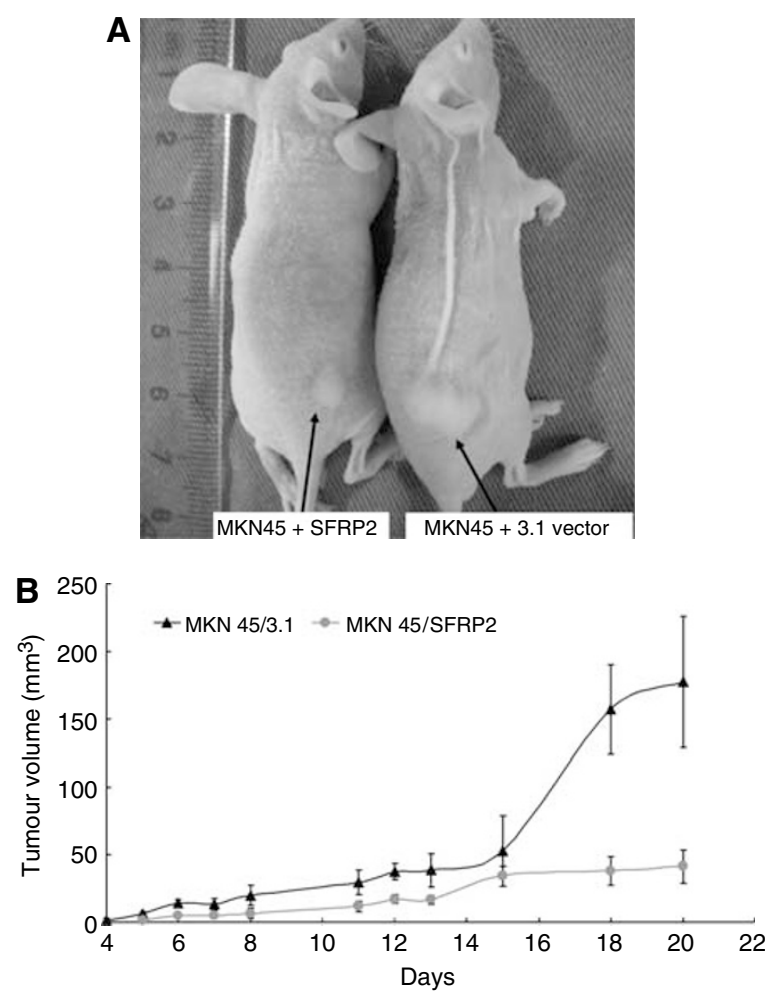

Figure 6 Secreted frizzled-related protein 2 (SFRP2) inhibits growth of tumours derived from MKN45 in vivo. (A) A representative picture of nude mice: at week 3 nude mice injected with MKN45/3.I and MKN45/SFRP2. (B) The tumour volume of vector (pcDNA3.I) transfected MKN45 cell in nude mice $(n=15)$ was indicated as mean tumour volume \pm s.d. $\left(\mathrm{mm}^{3}\right)$ Tumour mean volume of MKN45/SFRP2 mice was significantly smaller than the MKN45/3.I nude mice group $(* P<0.00$ I).

in intestinal metaplasia and $20 \%$ in adjacent non-cancer tissues. It thus appears that epigenetic alteration of SFRP2 is present in the pre-neoplastic stage even in patients without gastric cancer, which may be an important mechanism leading to malignant transformation. Since not all intestinal metaplasia (IM) will progress to gastric cancer and promoter hypermethylation is not detected in all gastric cancers, follow-up study is required to elucidate the prognostic value of detecting promoter hypermethylation in IM. Thus, detection of hypermethylation may have the potential of identifying individuals at risk for further histological progression.

The detection of SFRP2 methylated DNA in serum samples of gastric cancer patients further implies their potential diagnostic value in gastric cancer. Whilst we have previously shown that methylated DNA could also be detected in cancer patients' blood (Lee et al, 2002; Leung et al, 2005), it is therefore valuable to explore the possible application of SFRP2 as a serum molecular biomarker for detection of human gastric cancer. We found that SFRP2 was detected in $67 \%$ of serum samples of cancer patients. This is higher than the previous reports in which $44-55 \%$ of gastric cancer patients had methylation detected with the use of multiple markers (Koike et al, 2004; Leung et al, 2005). Our finding suggests that SFRP2 is a potential source of circulating methylated DNA. Studies with larger sample size are necessary to discern the early diagnostic and prognostic significance of detecting SFRP2 methylation in the serum of gastric cancer patients with different subtypes.

In conclusion, we found that SFRP2 is hypermethylated in gastric cancer. Although widely expressed in non-cancer samples, 
SFRP2 is silenced by methylation in gastric cancer samples. We further demonstrated that SFRP2 could act as a functional tumour suppressor gene in gastric cancer. The high detection rate of methylated SFRP2 in serum indicates that SFRP2 methylation has its diagnostic and therapeutic values in gastric cancer.

\section{ACKNOWLEDGEMENTS}

The work described in this paper was fully supported by a grant from the Research Grants Council of the Hong Kong Special Administrative Region (Project no. CUHK4473/05M).

\section{REFERENCES}

Chan MW, Wei SH, Wen P, Wang Z, Matei DE, Liu JC, Liyanarachchi S, Brown R, Nephew KP, Yan PS, Huang TH (2005) Hypermethylation of $18 \mathrm{~S}$ and $28 \mathrm{~S}$ ribosomal DNAs predicts progression-free survival in patients with ovarian cancer. Clin Cancer Res 11(20): 7376-7383

Fukui T, Kondo M, Ito G, Maeda O, Sato N, Yoshioka H, Yokoi K, Ueda Y, Shimokata K, Sekido Y (2005) Transcriptional silencing of secreted frizzled related protein 1 (SFRP 1) by promoter hypermethylation in non-small-cell lung cancer. Oncogene 24(41): 6323-6327

Heller RS, Dichmann DS, Jensen J, Miller C, Wong G, Madsen OD, Serup P (2002) Expression patterns of Wnts, Frizzleds, SFRPs, and misexpression in transgenic mice suggesting a role for Wnts in pancreas and foregut pattern formation. Dev Dyn 225(3): 260-270

Ilyas M (2005) Wnt signalling and the mechanistic basis of tumour development. $J$ Pathol 205(2): 130 - 144

Kang GH, Shim YH, Jung HY, Kim WH, Ro JY, Rhyu MG (2001) CpG island methylation in premalignant stages of gastric carcinoma. Cancer Res 61(7): $2847-2851$

Kirikoshi H, Sekihara H, Katoh M (2001) Expression profiles of 10 members of frizzled gene family in human gastric cancer. Int $J$ Oncol 19(4): $767-771$

Ko J, Ryu KS, Lee YH, Na DS, Kim YS, Oh YM, Kim IS, Kim JW (2002) Human secreted frizzled-related protein is down-regulated and induces apoptosis in human cervical cancer. Exp Cell Res 280(2): 280-287

Koike H, Ichikawa D, Ikoma H, Otsuji E, Kitamura K, Yamagishi H (2004) Comparison of methylation-specific polymerase chain reaction (MSP) with reverse transcriptase-polymerase chain reaction (RT-PCR) in peripheral blood of gastric cancer patients. J Surg Oncol 87(4): $182-186$

Lee TL, Leung WK, Chan MW, Ng EK, Tong JH, Lo KW, Chung SC, Sung JJ, To KF (2002) Detection of gene promoter hypermethylation in the tumor and serum of patients with gastric carcinoma. Clin Cancer Res 8(6): $1761-1766$

Leung WK, Man EP, Yu J, Go MY, To KF, Yamaoka Y, Cheng VY, Ng EK, Sung JJ (2006) Effects of Helicobacter pylori eradication on methylation status of E-cadherin gene in noncancerous stomach. Clin Cancer Res 12(10): $3216-3221$

Leung WK, To KF, Chu ES, Chan MW, Bai AH, Ng EK, Chan FK, Sung JJ (2005) Potential diagnostic and prognostic values of detecting promoter hypermethylation in the serum of patients with gastric cancer. $\mathrm{Br} J$ Cancer 92(12): 2190-2194

Leung WK, Yu J, Ng EK, To KF, Ma PK, Lee TL, Go MY, Chung SC, Sung JJ (2001) Concurrent hypermethylation of multiple tumor-related genes in gastric carcinoma and adjacent normal tissues. Cancer 91(12): 2294-2301

Marsit CJ, Karagas MR, Andrew A, Liu M, Danaee H, Schned AR, Nelson HH, Kelsey KT (2005) Epigenetic inactivation of SFRP genes and TP53 alteration act jointly as markers of invasive bladder cancer. Cancer Res 65(16): $7081-7085$

Marsit CJ, McClean MD, Furniss CS, Kelsey KT (2006) Epigenetic inactivation of the SFRP genes is associated with drinking, smoking and HPV in head and neck squamous cell carcinoma. Int J Cancer 119(8): $1761-1766$

Melkonyan HS, Chang WC, Shapiro JP, Mahadevappa M, Fitzpatrick PA, Kiefer MC, Tomei LD, Umansky SR (1997) SARPs: a family of secreted apoptosis-related proteins. Proc Natl Acad Sci USA 94(25): 13636-13641

Polakis P (2007) The many ways of Wnt in cancer. Curr Opin Genet Dev 17(1): $45-51$

Suzuki H, Gabrielson E, Chen W, Anbazhagan R, van Engeland M, Weijenberg MP, Herman JG, Baylin SB (2002) A genomic screen for genes upregulated by demethylation and histone deacetylase inhibition in human colorectal cancer. Nat Genet 31(2): 141-149

Suzuki H, Watkins DN, Jair KW, Schuebel KE, Markowitz SD, Chen WD, Pretlow TP, Yang B, Akiyama Y, Van Engeland M, Toyota M, Tokino T, Hinoda Y, Imai K, Herman JG, Baylin SB (2004) Epigenetic inactivation of SFRP genes allows constitutive WNT signaling in colorectal cancer. Nat Genet 36(4): $417-422$

Tamura G (2002) Genetic and epigenetic alterations of tumor suppressor and tumor-related genes in gastric cancer. Histol Histopathol 17(1): $323-329$

To KF, Chan MW, Leung WK, Yu J, Tong JH, Lee TL, Chan FK, Sung JJ (2001) Alterations of frizzled (FzE3) and secreted frizzled related protein (hSFRP) expression in gastric cancer. Life Sci 70(4): 483-489

To KF, Leung WK, Lee TL, Yu J, Tong JH, Chan MW, Ng EK, Chung SC, Sung JJ (2002) Promoter hypermethylation of tumor-related genes in gastric intestinal metaplasia of patients with and without gastric cancer. Int J Cancer 102(6): 623-628

Waki T, Tamura G, Tsuchiya T, Sato K, Nishizuka S, Motoyama T (2002) Promoter methylation status of E-cadherin, hMLH1, and p16 genes in nonneoplastic gastric epithelia. Am J Pathol 161(2): 399-403

Yagi MJ, Bekesi JG (1990) Evaluation of p-F-Phe-m-bis-(2-chloroethyl) amino-L-Phe-Met-ethoxy $\mathrm{HCl}$ against transplantable and spontaneous murine neoplasia. Cancer Chemother Pharmacol 26(3): 215-220

Yu J, Leung WK, Ebert MP, Leong RW, Tse PC, Chan MW, Bai AH, To KF, Malfertheiner P, Sung JJ (2003) Absence of cyclin D2 expression is associated with promoter hypermethylation in gastric cancer. $\mathrm{Br}$ J Cancer 88(10): 1560 - 1565

Zou H, Molina JR, Harrington JJ, Osborn NK, Klatt KK, Romero Y, Burgart LJ, Ahlquist DA (2005) Aberrant methylation of secreted frizzled-related protein genes in esophageal adenocarcinoma and Barrett's esophagus. Int J Cancer 116(4): 584-591 